\title{
Chemical Reactor Modelling (CRN) of a Reverse Flow Combustor
}

\author{
Gurkan Sarikaya, Gokhan Varol, Onur Tuncer \\ Istanbul Technical University (ITU) \\ Maslak, Sariyer/İstanbul, Turkey \\ sarikayagu@itu.edu.tr; varolgo@itu.edu.tr; tuncero@itu.edu.tr
}

\section{Extended Abstract}

As today demands, the gas turbine engines should be able to burn fuels efficiently while keeping emissions at a minimum level. In this study, a 1/13 sector of a reverse flow annular combustor, intended to be used in a $1000 \mathrm{hp}$ turboshaft helicopter engine, is investigated to predict the $\mathrm{NOx}$ and $\mathrm{CO}$ emissions by modelling chemical reactor network (CRN) according to results of CFD simulations.

The real geometrical dimensions of combustor were used without any scaling factor to create computational domain. The 3D generated mesh has roughly 10 million unstructured polyhedral elements.

The CFD simulations were performed in order to determine the reacting flow characteristics of combustor by using FLUENT ${ }^{\circledR}$ software. The Shear-Stress Transport (SST) k- $\omega$ model was used for turbulent flow coupled with the steady diffusion flamelet combustion model for reacting analysis. The flame-turbulence interaction was described by combining flamelet concept with the Probability Density Function (PDF) closure method. With this concept, the thermochemical properties of an adiabatic turbulent flame can be defined by using the mixture fraction and the scalar dissipation rate at stoichiometric mixture fraction. The $\beta$-PDF method was used to generate a look-up table database for turbulent combustion. For chemical reaction, n-heptane fuel mechanism which was derived by the University of California San Diego [1], is used for chemical reaction process. It has 58 species and 325 reactions.

Velocity and temperature profiles obtained from reactive CFD analysis for the combustor are determinative in CRN modelling of re-circulation, primary, secondary, and dilution zones.

The CRN modelling studies in literature are usually done for axisymmetric combustors which have the same temperature and velocity profiles along the radial direction and modelled according to 2D CFD analysis results. The modelling combustor by CRN method was firstly described by Bragg [2]. Novesselov [3] also modelled a gas turbine combustor as an association of a perfectly stirred reactor (PSR) followed by a plug flow reactor (PFR) in 2D plane. Sam Ghazi-Hesami [4] used a different way by using a flow field discretization method to construct CRN model in 3D dimensional combustor. Falcitelli et.al. [5] developed an algorithm for extracting CRN model from CFD simulation of an industrial combustion system.

While in this study, to overcome the complexity of 3D results, a different method was applied for 3D combustor with having variable speed and temperature profile along the z-direction. In this method, CFD result of 3D combustor was converted to the $2 \mathrm{D}$ plane by using mass weighted averaged technique. Based on the converted results in $2 \mathrm{D}$ plane, reactor network model was created.

In the $3 \mathrm{D}$ to $2 \mathrm{D}$ conversion, firstly, 2D slices were cut with equal distance along the direction of $\mathrm{z}$ axis. Since the mesh structure in each obtained slice is different, the computational nodes in each slice are also located at different coordinates. For this reason, the results in each obtained slice were placed into a newly generated and regular meshed surface in TECPLOT ${ }^{\circledR}$ software by using inverse-distance interpolation method. This method is that takes the average values in data points of the source zone to different points in target zone [6].

The mass weighted averaged of all the quantities such as temperature, density, and velocity at the same nodes in each slice was computed and transferred to a one piece of 2D plane.

Analysis of numerical results and mass averaged computation has allowed the identification of conditions in which the reaction zone of combustor can be modelled as a PSR and PFR in 2D dimensional surface by using CHEMKIN® software. In this study, temperature gradients are determinative for reactor locations. Primary and secondary zones are modelled as PSR and dilution zone which in no radial flow motion, is modelled as PFR. Mass flow splits between each adjacent zone are calculated by using velocity profile. 
Consequently, a MATLAB tool that will enable to user to identify the reactor zones with mouse is currently under the construction. By using this tool, mass flow splits and zone areas are going to provide input to CHEMKIN® software.

Finally, CRN model will be coupled with detailed chemical reaction mechanism to predict the NOx and CO emissions of reverse flow combustor.

\section{Reference}

[1] "Chemical-Kinetic Mechanisms for Combustion Applications," Mechanical and Aerospace Engineering (Combustion Research), University of California at San Diego. Available: http://combustion.ucsd.edu.

[2] S. L. Bragg, "Application reaction rate theory to combustion chamber analysis," Aeronautical Research Council Pub., ARC 16170, pp. 1629-1633, 1953.

[3] I. V. Novosselov, "Chemical reactor network modeling of combustion systems", Ph.D. dissertation, University of Washington, Seattle.

[4] S. Ghazi-Hesami, "Cost Effective Emissions and Minor Species Predictions via Coupling of Computational Fluid Dynamics and Chemical Reactor Network Analysis," Master's thesis, Concordia Univ., Montreal, 2009.

[5] M. Falcitelli, L. Tognotti, S. Pasini, "An Algorithm for Extracting Chemical Reactor Network Models from CFD Simulation of Industrial Combustion Systems," Combustion Science and Technology, vol. 174, no. 11-12, 2002.

[6] 2016. Tecplot 360 User's Manual [Online]. Available:

http://download.tecplot.com/360/current/360_users_manual.pdf. 